\title{
Namenformen als Schlüssel zur Geschichte Win-win-Effekt für Geschichts- und Sprachforschung
}

\author{
von \\ KARLHEINZ HENGST
}

\begin{abstract}
Den Anstoß zu diesem Beitrag und die entsprechenden Fakten aus der historischen Tradierung zur Geschichte der Stadt Leipzig hat der Ende 2015 im Leipziger Universitätsverlag erschienene erste Band der umfangreichen „Geschichte der Stadt Leipzig“ unter verantwortlicher Gesamtleitung von Enno Bünz als Inhaber des Lehrstuhls für Landesgeschichte an der Universität Leipzig gegeben. Mit der nachfolgenden Darstellung lässt sich verdeutlichen, dass Landesgeschichte und Sprachgeschichte bei gegenseitiger Nutzung ihrer Forschungsergebnisse stets neuen Gewinn auf beiden Seiten verzeichnen können.

Historiker stützen sich darauf und vertrauen dem, was in Urkunden oder erzählenden Quellen an Fakten mitgeteilt wird. Das kann auch nicht anders sein und gilt expressis verbis auch für das ad 1015 erstmals bei Thietmar von Merseburg mit der Form Libzi erwähnte Leipzig und sein daher 2015 gefeiertes Jubiläum. Es gilt dies ganz ausdrücklich auch für die Sprachforschung. Sie orientiert sich an den ältesten sprachlichen Zeugnissen in Urkunden und erzählenden Quellen. Und sie vertraut den Archivaren sowie Historikern als Editoren von Urkunden sowie Interpreten von nur in Abschriften aus späteren Jahrhunderten erhalten gebliebenen älteren Originalurkunden. Unter Nutzung der aus sprachhistorischer Forschung gewonnenen Erkenntnisse zur lautlichen Entwicklung und damit auch zur Veränderung von Eigennamen aus Jahrhunderten lange vor der Verschriftlichung von Texten mit ersten Namenaufzeichnungen ist es möglich geworden, Eigennamen als Geschichtsquellen auch zur Frühgeschichte im heutigen deutschen Sprachraum zu Aussagen zu führen. Dazu besteht eigentlich auch - ganz besonders in Sachsen und Thüringen, aber auch weit darüber hinaus - zwischen Historikern und Sprachforschern Konsens. Davon zeugen die von beiden Seiten jeweils ausgesprochenen Einladungen zur Mitwirkung an Projekten und größeren Vorhaben. ${ }^{1}$ Institutionell wird diese Kooperation z. B. auch dokumentiert durch die mehrfache Vertretung der Sprachwissenschaft in der „Historischen Kommission" bei der Sächsischen Akademie der Wissenschaften zu Leipzig. Rahmen und Bedingungen für eine gedeihliche wechselseitige Nutzung von Forschungsergebnissen sind also gegeben und ausreichend vorhanden. Die historische Sprachforschung hat den ungemeinen Vorteil, dass sie Eigennamen in zumeist lateinisch geschriebenen Texten als Zeugen und Denkmäler aus oftmals weit vor den ersten schriftlichen Aufzeichnungen liegenden Sprachverhältnissen nutzen kann. Die Forschungsgeschichte hat
\end{abstract}

1 Das trifft auch voll zu für das neue Geschichtswerk zur Stadt Leipzig mit einem den Forschungsstand zum Namen Leipzig zusammenfassenden Beitrag des Sprach- und Siedlungshistorikers Hans Walther, Der Name Leipzig in seiner Herkunft und Entwicklung, in: Enno Bünz (Hg.), Geschichte der Stadt Leipzig, Bd. 1: Von den Anfängen bis zur Reformation, Leipzig 2015, S. 73-76 und gilt auch für den ebenda gemeinsam mit dem Archäologen Thomas Westphalen gebotenen Überblick zu den slawischen Siedlungen und ihren Namen: Thomas Westphalen/Christian Zschieschang, Die Slawen im Leipziger Raum. Archäologie und Ortsnamen, in: ebd., S. 57-72, hier S. 60-72. 
klar erwiesen, dass die Eigennamen trotz der für manchen Betrachter oftmals recht undurchsichtig bis willkürlich erscheinenden Schreibweisen bei der gewissenhaften Analyse keinesfalls diesem Eindruck entsprechen. Es sind vielmehr der jeweiligen Zeit gemäße Regularitäten auch bei der schriftlichen Fixierung von Namen aus fremden Sprachen befolgt worden. Das musste auch so sein, denn die Namen dienten sowohl in der mündlichen Kommunikation als auch bei ihrer Verschriftlichung in Urkunden immer auch der Sicherung von zweifelsfreier Identifizierung. Das galt in ganz besonderem Maße auch für Gebiete mit Bewohnern unterschiedlicher Ethnien und Sprachen, also auch für den ab dem 10. Jahrhundert ins Fränkische Reich einbezogenen Raum östlich der Saale. Subtile sprachwissenschaftliche Forschungsarbeit hat in der zweiten Hälfte des 20. Jahrhunderts für das Gebiet der slawisch-deutschen Sprachkontakte aus den überlieferten Onymen eine Vielzahl an historischen Fakten zur Kulturgeschichte der Sprachträger beider Ethnien in Nachschlagewerken zur Nutzung bereitstellen können. Die Linguistik konnte mittels komplexer sowie verfeinerter Forschungsmethodik Rückblicke bis in die Zeit der ersten germanisch-slawischen Kontakte in der slawischen Landnahmezeit ebenso ermöglichen wie solche zu Leben und Umweltbewertung durch die Slawen etwa vom 8. Jahrhundert an und die Rezeption sowie Tradierung der Namen durch deutsche Sprecher vor allem seit dem 10. Jahrhundert. Das gilt auch für den Leipzig-Namen und damit für die aus diesem für die frühe Geschichte von Geografie und Leben der Menschen im Raum des heutigen Leipzig gewonnenen Einblicke.

Da fällt aber nun andererseits doch auf, wenn von Historikerseite wiederkehrend erwähnt wird, dass „über die Geschichte Leipzigs vor 1015 nichts bekannt ist“. ${ }^{2}$ Nun darf man diese Aussage nicht aus dem Zusammenhang reißen und zu einer verzerrten Interpretation verwenden. Diese Feststellung ist nämlich voll zutreffend im Zusammenhang mit Betrachtungen zur frühen Kirchenorganisation von Leipzig. Und genau darum ging es in der Publikation. Andererseits wirken die Worte, dass vor 1015 zur Geschichte von Leipzig $\mathrm{n}$ i c h t s bekannt ist, doch zugleich als eine sehr generelle Aussage oder können zumindest so verstanden werden. Dieser Eindruck verstärkt sich noch bei der Lektüre im ersten Band der neuen und umfangreichen Geschichte der Stadt Leipzig. ${ }^{3}$ Wiederum im Hinblick auf die kirchenorganisatorische Zugehörigkeit ist zu lesen: „Falls der Ort Leipzig Ende 968 schon existiert haben sollte, wäre er kirchenorganisatorisch dem Bistum Merseburg zugewiesen worden."4 Und der vorsichtige Zweifel wird sogar nochmals wiederholt zu einem späteren Jahr mit „falls es den Ort Leipzig 981 schon gegeben haben sollte“. ${ }^{5}$ Mit ausdrücklicher Beschränkung auf die in Text gefassten Angaben ist die Vorsicht und Zurückhaltung des Mittelalterhistorikers Wolfgang Huschner (ebenfalls Universität Leipzig) durchaus verständlich. Andererseits veranlassen die Vorbehalte und dahinter sichtbar werdenden Bedenken zugleich doch zu weiterem Nachfragen, ob es denn wirklich keine Indizien in den Texten zu einem möglichen Ort Leipzig vor 968 gibt.

Nun erscheint es wahrscheinlich verwunderlich oder gar anmaßend, kurz nach dem Erscheinen eines so gründlich gearbeiteten und von namhaften Historikern ge-

2 Enno Bünz, Mehr als die berühmtesten Pfarrkirchen der Welt. Das kirchliche Leipzig vor der Reformation, in: Sächsische Heimatblätter 61 (2015), H. 2, S. 107-121, hier S. 109.

3 Bünz, Geschichte der Stadt Leipzig 1 (wie Anm. 1).

4 Wolfgang Huschner, Die kirchenorganisatorischen Zuordnungen des Ortes Leipzig. Gründung, Aufhebung und Wiedererrichtung des Bistums Merseburg (962-1024), in: Bünz, Geschichte der Stadt Leipzig 1 (wie Anm. 1), S. 90-109, hier S. 97.

5 Ebd., S. 99. 
stalteten Werkes eine Wortmeldung aus einer der Geschichtswissenschaft benachbarten Disziplin abgeben zu wollen. Noch dazu nicht aus der Archäologie mit ihren immerhin handfesten Fundbelegen zur Frühzeit, sondern aus sprachhistorischer Sicht, also mit Blick auf die Sprachgeschichte. Dennoch sei es wenigstens versucht. In der sprachgeschichtlichen Forschung ist es immerhin in den letzten Jahrzehnten gelungen, aus früh überlieferten sprachlichen Formen a) Schlüsse zur Zeit ihrer Prägung und Entstehung sowie damit auch b) Aussagen zu Alter und Charakter des jeweiligen Referenzobjektes zu gewinnen. Da die älteste urkundliche Tradierung - genau wie auch bei der Chronik von Thietmar - in lateinischer Sprache erfolgte, sind jene Worte in den historischen Dokumenten von besonderem Wert, die aus der gesprochenen Sprache ihrer Zeit übernommen wurden und in die Aufzeichnungen eingeflossen sind. Das sind vorwiegend die Eigennamen von Urkundenaussteller und Urkundenempfänger, von Zeugen sowie - und das ist hier nachfolgend von Interesse - von erwähnten Örtlichkeiten und Angaben zu ihrer Lage.

Aufgrund der von Thietmar ad 1015 gemachten Angaben in urbe Libzi vocata und aecclesias in Libzi et in [...] ist nun tatsächlich mehr erkennbar als nur die Existenz von Burg und Kirche im damaligen Ort. Für den mit den sprachlichen Verhältnissen in jener Zeit und aufgrund langjähriger Forschungen zur Wiedergabe von Namen in der urkundlichen Tradierung tätigen Sprachhistoriker sind das im Einzelnen folgende Fakten:

1. Libzi ist eine zweifelsfrei aus dem Slawischen übernommene Sprachform. Sie beruht auf einer noch in gemeinslawischer Zeit, also im 9./10. Jahrhundert, gebräuchlichen Form, die sich rekonstruieren lässt (daher mit * versehen) als * Libbci (gesprochen etwa [libĭtsi], wobei das Ǐ superkurz gesprochen wurde).

2. Das im Anschluss an die Namenbasis * Lib-verwendete Suffix - bci kennzeichnete zunächst eine Personengruppe. Diese bedeutete etwa ,die ,Hageren', also die ,Familie/Leute des Hageren'. Diese Benennung wurde zur Ortsangabe, lässt also auf einen ursprünglich kleinen Ort schließen, der nach einer persönlichen Auffälligkeit eines Bewohners benannt wurde.

3. Um die Jahrtausendwende n. Chr. sind im slawischen Sprachraum die ultrakurzen Vokale (also hier das $\breve{i}$ ) in bestimmten Positionen geschwunden bzw. verändert worden, was ganz lautgerecht zu der bei Thietmar fixierten Form Libz- in der Gestalt von Libzi [libtsi] führte.

4. Die Basis Lib-ist - nach dem neuesten Forschungsstand - inzwischen also aus dem Slawischen erklärbar.6 ${ }^{6}$ Die Namengebung ist auf jeden Fall lange Zeit vor Thietmars Aufzeichnung erfolgt. Spätestens im 9. Jahrhundert muss es daher eine kleine slawische Ansiedlung gegeben haben. Und wenn dort eine urbs ad 1115 sowie eine aecclesia ad 1017 von Thietmar bezeugt werden, ist sehr wahrscheinlich von deutscher Seite nach 930 ein - aus sicher mancherlei Gründen - als bedeutsam und wichtig angesehener Ort befestigt und weiter ausgestattet worden.

Als Ergebnis können wir also bisher festhalten: Es hätte keinen slawischen Ortsnamen in der Aufzeichnung mit der Form Libzi gegeben, wenn nicht auch eine damit benannte slawische Siedlung vorhanden gewesen wäre. Diese ist von den slawischen Bewohnern, die in benachbarten Niederlassungen lebten, im 9./10. Jahrhundert mit dem Namen *Libbci von anderen Orten unterschieden worden. Und dieser Name ist auch im 11. und 12. Jahrhundert von den bis dahin die Mehrheit der Bevölkerung

6 Vgl. dazu die Beiträge von Bernd Koenitz, Karlheinz Hengst und Walter Wenzel in: Namenkundliche Informationen/Journal of Onomastics 107/108 (2016), S. 441-481. 
bildenden slawischen Bewohnern ${ }^{7}$ beibehalten worden, aber in dieser Zeit nicht mehr als [libĭtsi] gesprochen worden, sondern lautete infolge der Veränderung bei den ultrakurzen Vokalen [libtsi] und wurde auch so von den Deutschen gehört und entsprechend niedergeschrieben. Das lässt sich „urkundlich“ auch mit weiteren Belegen beweisen:

Noch 1185 werden die slawischen Adligen Albertus de $L i b z$ et frater suus Burzlaus genannt. ${ }^{8}$ Die hier absichtlich gesperrt gesetzte Form Libz gibt zugleich zu erkennen, dass der Name nun der inzwischen in altsorbischer Zeit eingetretenen Veränderung $\mathrm{zu}{ }^{*} L i b$ 'c folgend (gesprochen [libts] mit palatalem /b/) die neuere Gebrauchsform ausweist. Die urkundliche Schreibweise entspricht der bei Thietmar. Auf eine Wiederholung der ausführlichen Darstellung zu den Gründen für die Grafien Libiz (Lehnform mit Bewahrung der slawischen Lautung vom 9./10. Jahrhundert) und zum anderen nur Libz (Entlehnung gemäß der nach 1000 n. Chr. gesprochenen altsorbischen Form) wird hier verzichtet. ${ }^{9}$ Darauf wird aber gleich nochmals kurz zurückzukommen sein, um den Wechsel von Formen in den Aufzeichnungen verständlich zu machen.

Die um 1150 geschriebenen Pegauer Annalen geben nämlich erstaunlicherweise wiederum ältere Formen mit folgenden Belegen zu erkennen: usque Libiz, Libiz oppi$d u m .{ }^{10}$ Das mag verwirrend erscheinen, vielleicht auch den Eindruck erwecken, dass die Schreibungen gar willkürlich erfolgten - und am Ende vom Betrachter aus heutiger Zeit als nicht gerade zuverlässig bewertet werden. Doch die Tatsachen sprechen gegen ein solches Bild. Allerdings ist dazu unbedingt zu beachten, dass östlich der Saale seit dem 10. Jahrhundert nicht nur die slawischen Bewohner, sondern auch die deutsche Obrigkeit die slawischen Namen verwendeten. Nach der militärischen Besetzung und mit der Einrichtung von entsprechenden Stützpunkten respektive Verwaltungszentren und der Markenbildung ging die Übernahme der slawischen geografischen Namen als völlig normaler Prozess einher. Die bereits ab der Eroberung ins damalige gesprochene Althochdeutsch oder aber Altsächsisch aufgenommenen Namen reflektieren die zu jener Zeit noch dialektalen gemeinslawischen bzw. späturslawischen Sprachverhältnisse. Die ultrakurzen Vokale wurden im 10. Jahrhundert noch gesprochen und daher auch von den deutschen Sprachträgern gehört sowie entsprechend übernommen. Einmal nun in althochdeutscher Form in der Lautung [Libitsi] < slawisch *Libbci gebräuchlich geworden, wurde auch diese Lehnform nun beibehalten. Diese Form wurde in der Kommunikation sowohl mit den einheimischen Slawen als auch innerhalb der deutschen Sprechergruppen so verwendet. Die Verständigung war damit

7 Diese slawische Majorität ist noch heute leicht ablesbar auf dem Kartenbild mit den slawisch geprägten Siedlungsnamen im Leipziger Raum; vgl. zuletzt WestPHALEN/ Zschieschang, Die Slawen im Leipziger Raum (wie Anm. 1), S. 57-72, insbesondere auch Karte 4, S. 65 sowie die Ortsnamenübersicht S. 68-72.

8 Codex diplomaticus Saxoniae regiae, I. Hauptteil, Bd. 2 (im Folgenden: CDS I/2): Отто Posse (Hg.), Die Urkunden der Markgrafen von Meißen und Landgrafen von Thüringen 1100-1195, Leipzig 1889, Nr. 510.

9 Vgl. dazu Karlheinz Hengst, Der Name Leipzig als sprachliches Denkmal aus frühgeschichtlicher Zeit, in: Leipziger Stadtgeschichte. Jahrbuch 2010, S. 9-20, insbesondere S. 14-16.

10 Vgl. Hans Walther, Zum Namen der Stadt Leipzig, in: Ernst Eichler/Hans Walther, Alt-Leipzig und das Leipziger Land. Ein historisch-geographisches Namenbuch zur Frühzeit im Elster-Pleißen-Land im Rahmen der Sprach- und Siedlungsgeschichte (Onomastica Lipsiensia. Leipziger Untersuchungen zur Namenforschung 7), Leipzig 2010, S. 129. 
sowohl in der interethnischen als auch in der binnenethnischen deutschen Kommunikation garantiert.

Mit dem Übergang zum mittelhochdeutschen Sprachusus ab ca. rund 1050 vollzog sich eine Abschwächung der Auslautvokale bis hin zu ihrem völligen Schwund. Daher bringen die Pegauer Annalen um 1150 einerseits mit Libiz das Lautbild der Lehnform aus dem 10. Jahrhundert nochmals zum Vorschein. Die Endungslosigkeit, also der Wegfall des auslautenden $-i$, kann bereits den Verlust des im 10. Jahrhundert vorhandenen ursprünglichen Auslautvokals im Deutschen reflektieren, beruht aber wohl eher auf der Wiedergabe einer in der slawischen alltäglichen mündlichen Kommunikation im Syntagma verwendeten endungslosen Genitiv-Plural-Form. Damit bieten die Pegauer Annalen offensichtlich die in der Kanzlei beibehaltene Lehnform Libiz aus dem 10. Jahrhundert - nicht aber die alltagssprachliche neuere Form des 11./12. Jahrhunderts Libz (Genitiv Plural) oder Libzi (Nominativ).

$1185 \mathrm{Libz}$ ist ganz normal die Wiedergabe einer sich ab dem 11. Jahrhundert zeigenden Neuentlehnung aus dem Slawischen in den spätalthochdeutschen bzw. frühmittelhochdeutschen Sprachgebrauch gemäß der neuen slawischen Sprechsituation mit dem Schwund der ultrakurzen Vokale in bestimmten Positionen innerhalb der Sprachformen. Die Neuentlehnung aus dem jetzt gesprochenen frühaltsorbischen *Lib'c [lib'ts] - urkundlich geschrieben <Libz> sowohl bei Thietmar als Kenner der Sprache vor Ort als auch 1185 bei Zeugenbenennung - entspricht voll und ganz den in jener Zeit geltenden sprachlichen Verhältnissen.

Die Grafien sind absolut korrekt und an keiner Stelle willkürlich oder merkwürdig erfolgt. Im 11./12. Jahrhundert haben wir ein Nebeneinander von älterer Lehnform Libiz und jüngerer Lehnform $\mathrm{Libz}$ in der urkundlichen Überlieferung als Folge von zwei gebräuchlichen Lehnnamenformen zu beachten.

Hier kann nun neuerdings auf eine zweite frühe Nennung einer Libiz-Form in einer Urkunde aus dem 11. Jahrhundert noch zusätzlich als sprachgeschichtliches Beweismittel aufmerksam gemacht werden. Es handelt sich um ein Diplom von Kaiser Heinrich III. Es liegt aber nicht mehr im Original, sondern nur in einer Abschrift vor.

Der Landeshistoriker Enno Bünz hat gegenüber bisherigen Annahmen zu dieser aus dem Jahr 1050 stammenden Urkunde eine ganz wichtige Klarstellung zu Inhalt und Form des Textes vorgenommen. Der Kaiser beurkundet in Wurzen eine Schenkung an das Bistum Merseburg. Diese betrifft villam Nuwindorph dictam in comitatu Wilhelmi marchionis in pago Szudici in burgvardo Libizken sitam. ${ }^{11}$ Enno Bünz hat mit Nachdruck darauf aufmerksam gemacht, dass diese zwar nur in einem Kopialbuch von 1427 bis 1430 enthaltene Urkunde keine Fälschung aus späterer Zeit ist und voll inhaltlich eine zuverlässige Kopie darstellt. ${ }^{12}$ Die Richtigstellung endet mit folgenden Worten: „An der Echtheit der Urkunde besteht kein Zweifel."13 Dies ist für die Sprach- respektive Namenforschung besonders wichtig, da selbst der mit dem Bistum Merseburg sehr vertraute Historiker und Germanist Hans Walther davon ausging, dass es sich bei der Urkunde um eine Fälschung aus dem 15. Jahrhundert handle. Die neu gewonnene Erkenntnis hat nun durchaus ihre Auswirkungen für die Namenforschung.

11 Paul Fridolin Kehr (Bearb.), Urkundenbuch des Hochstifts Merseburg (Geschichtsquellen der Provinz Sachsen 36), Teil I: 962-1357, Halle/Saale 1899, Nr. 71.

12 Vgl. dazu Enno Bünz, Herrschaftliche Strukturen. Die ottonische Markenorganisation zwischen Saale und Elbe, in: Bünz, Geschichte der Stadt Leipzig 1 (wie Anm. 1), S. 7989, hier S. 85, 89.

13 Ebd., S. 799, Anm. 51. Von der historischen Sprachforschung wurde die Urkunde bisher leider als Fälschung aus dem 15. Jahrhundert angesehen. 
Diese Urkunde enthält die klare Bezeugung des Burgwards Leipzig im 11. Jahrhundert mit der Angabe in burgvardo Libizken. Wiederum ist daraus aber weit mehr als nur der Nachweis für die Existenz des Burgwards mit seiner Benennung nach einem anzunehmenden Zentralort zu entnehmen. Allein die Form in burgvardo Libizken bietet dem Sprachhistoriker unter slavistischem sowie germanistischem Aspekt zugleich noch folgende Fakten:

1. Zunächst ist erneut die Basis Lib-mit dem bisher eindeutig als älter erkannten Libiz- in der Form Libizken einwandfrei bezeugt. Damit ist klar, dass auch der Burgwardname auf einem älteren und in slawischer Zeit gebrauchten Namen beruht. An der Endung -en ist die Verwendung des Burgwardnamens im deutschen Sprachgebrauch - also damals in spätalthochdeutscher oder altsächsischer $=$ altniederdeutscher Sprache - ohne besondere Mühe ablesbar.

2. Die Form in [...] Libizken aus dem Jahr 1050 im deutschen Sprachgebrauch ist auf eine slawische Ausgangsform rückführbar, die noch im 10. Jahrhundert *Libbsk-, in der vollen Form am ehesten *Libbskъ [*grodъ ,Burg"] oder *Libbsko [*sedto] gelautet haben dürfte bzw. gelautet haben muss.

3. Mit in burgvardo Libizken liegt zugleich der Nachweis vor, dass der slawische Name noch vor der Jahrtausendwende in den deutschen Sprachgebrauch fest übernommen wurde. Zum einen wurde die slawische Nachsilbe (Suffix) -bsk-völlig lautgerecht mit <izk $>$ in der Niederschrift wiedergegeben. ${ }^{14}$ Zum anderen ist das späturslawische ultrakurze /b/ noch als [i] gehört worden und somit auch in den deutschen Sprachgebrauch übernommen worden. Die Rekonstruktion einer späturslawischen Form des Burgwardnamens *Libssk- als ursprünglich maskuline oder neutrale Form ist damit ebenso zweifelsfrei gesichert wie die Echtheit der Namensform in der Urkunde von 1050 selbst.

4. Die in dem Syntagma in burgvardo Libizken nun auftretende und scheinbar störende Endung -en beruht in der Aufzeichnung auf deutschem Einfluss. Es liegt hier ein Beispiel für die Integration des slawisch gebildeten Namens und seine Verwendung als Burgwardname im althochdeutschen Sprachgebrauch aus dem 10. Jahrhundert vor. Im Urkundentext hat der bei der deutschen Obrigkeit (Kanzlei von König bzw. Kaiser sowie Markgraf) offenbar sehr geläufige slawische Name als Name für den Burgward seinen Niederschlag gefunden, und zwar so regelrecht, dass er der Syntax des Textes entsprechend die in jener Zeit im Althochdeutschen im Singular bei bestimmten Substantiven vorkommende Dativ- bzw. Lokativendung -en erhielt. Diese Deklinationsform spricht für die 1050 offenbar schon lange im deutschsprachigen Verwaltungsbereich geläufige Lehnform als Burgwardname. Schwerer zu entscheiden ist, welches grammatische Vorbild gewirkt hat. Es kann sich um eine im Vergleich mit deutschen Insassennamen ganz analog gewählte lokativische Kasusendung -en handeln. ${ }^{15}$ Es kann aber vielleicht auch ein anderes Modell gewirkt haben. Es kann dabei slawisches *Libbsk-als entlehntes althochdeutsch gesprochenes [*libitsk-] an althochdeutsche Substantive wie herza ,Herz" oder hano "Hahn' angeschlossen und entsprechend dem althochdeutschen Dativ Singular herzen bzw. hanen mit der regelgemäßen Endung -en versehen worden

14 Auf den sprachgeschichtlichen Hintergrund für diese Grafie wird hier aus Raumgründen nicht näher eingegangen.

15 Für die Diskussion dieser Möglichkeit mit entsprechenden Hinweisen auf vergleichbare deutsche Namen danke ich dem Germanisten und Sprachforscher Prof. Dr. Albrecht Greule von der Universität Regensburg. Er vermerkte ausdrücklich: „Wie dem auch sei, der Fall Libizk-en markiert einen interessanten (frühen) Schritt hin zu einem allgemeinen toponymischen Suffix /-en/ im Deutschen“ (brieflich am 30. Dezember 2015). 
sein. Anders formuliert: Es wird also die den lateinischen Ablativ Singular als Lokativform in burgvardo fortsetzende Ortskasusform auch in der Namensform Libizken ganz adäquat deutlich, nur in dem Lehnnamen nicht mit lateinischer, sondern eben mit deutscher Kasusendung. Das Motiv bzw. der Beweggrund zur Verwendung gerade dieser Kasusform durch den Notar ist in der Rückschau nicht mehr eindeutig bestimmbar. Sicher aber ist, dass es sich um eine alte deutsche Deklinationsendung an einem Lehnnamen handelt, der wohl schon als ganz eingedeutscht empfunden wurde.

5. Die 1050 bezeugte Form des Burgwardnamens liegt uns nur in dieser deklinierten Form ganz einmalig aus dem deutschen Sprachgebrauch vor. Es lässt sich ein gesprochener althochdeutscher Nominativ ["Libitsk] regelkonform rekonstruieren. Damit ist der slawische Name *Libbsk- als Grundlage für den Namen des unter Otto I. eingerichteten Burgwards erwiesen. Und die aufgezeichnete Form in [...] Libizken belegt zugleich, dass der Burgward im Elster-Pleiße-Gebiet auf einer slawischen Form v o r dem 11. Jahrhundert für den Burgwardort beruht. Fest steht mit Gewissheit, dass er im 10. Jahrhundert bereits in den deutschen herrschaftlichen Sprachgebrauch übernommen worden ist und auch nach deutschem Sprachusus verwendet wurde. Es hat also im 10. Jahrhundert einen deutschen Lehnnamen Libizk- gegeben. ${ }^{16}$ Beim Übergang zur mittelhochdeutschen Zeit ist $\mathrm{ab}$ dem 11. Jahrhundert auch mit Verlust des Endungsvokals und einer Verkürzung des Lehnnamens zu Libizk zu rechnen.

6. Der Burgwardname hat nach der Jahrtausendwende in altsorbischer Zeit nach dem Schwund des ultrakurzen $/ \mathrm{i} /$ in der zweiten bzw. vorletzten Silbe die Form *Lib'sk-17 angenommen. Diese ist allmählich und in Anklang an slawisch lipa ,Linde“ als *Lip'sk-gesprochen worden. Auch diese Form ist ins Deutsche übernommen worden. Sie hat mit Reduzierung des Auslautvokals in mittelhochdeutscher Zeit die ab Ausgang des 12. Jahrhunderts auftretende Lehnform mit den Schreibungen Lipzc oder Lipzk (gesprochen jeweils [liptsk]) ergeben. Nach wie vor also systemhaft und lautgerecht mit $<z>$ zur Wiedergabe des slawischen /s/.

Mit diesen Ausführungen ergibt sich nun aber ein Widerspruch zu den Angaben von Historikerseite zu der Urkunde von 1050. Bei Enno Bünz heißt es nämlich: „Das Diplom ist nur abschriftlich [...] überliefert; dies erklärt die ungewöhnliche Ortsnamenform ,Libizken', die nicht in das 11. Jahrhundert passt, sondern von dem Abschreiber des späten Mittelalters unwillkürlich an den Sprachstand seiner Zeit angepasst wurde." 18 Die in der urkundlichen Überlieferung einmalige Form Libizken ist daher eben auch ausdrücklich als „ungewöhnliche Ortsnamenform“19 bezeichnet worden. ${ }^{20}$

16 In dem ermittelten Lehnnamen Libizk- hat ein in althochdeutscher Zeit vorhandener Auslautvokal in mittelhochdeutscher Zeit - also etwa ab 2. Hälfte 11. Jahrhundert - eine Abschwächung erfahren und ist schließlich ganz geschwunden, genau wie z. B. bei der Entwicklung von althochdeutsch herza zu mittelhochdeutsch berze und herz.

$17 b$ ' beruht auf rein sprachwissenschaftlich begründeter Schreibweise und zeigt als Folge des geschwundenen hellen Vokals noch die palatale Färbung des slawischen Phonems $|b|$ an.

18 BüNZ, Herrschaftliche Strukturen (wie Anm. 12), S. 89.

19 Ebd., S. 85.

20 Ein Blick in das Register des dreibändigen Urkundenbuchs der Stadt Leipzig bestätigt dies. In Band 3 sind auf S. 473 diverse Schreibungen zu finden, aber nicht eine einzige hat $-e n$ oder eine andere geschlossene Silbe nach $-k-$. Auch die Auflistung urkundlicher Belege bei Walther, Zum Namen der Stadt Leipzig (wie Anm. 10), S. 129 f. hat nur einmalig 1050 Libizken. 
Dieser anscheinende Widerspruch lässt sich jedoch leicht beseitigen. Dabei spielt die frühe Erwähnung als Name für den Burgward eine Rolle. Wichtig ist zugleich die Beachtung der sprachhistorischen Gegebenheiten, die dem Historiker so nicht bekannt sein können, sodass er eine ganz andere Schlussfolgerung zieht.

$\mathrm{Da}$ ist zunächst nochmals festzustellen, dass es auch in der späteren Überlieferung des Namens Leipzig keine vergleichbare Schreibung mit dem Auslaut $<$ ken $>$ gibt. Die Annahme einer Modernisierung des Namens durch den Kopisten scheidet somit mangels ähnlicher und irgendwie vergleichbarer Grafien aus der Überlieferung zu dem Namen Leipzig aus. Im Gegenteil, mit der sprachgeschichtlichen Analyse von in burgvardo Libizken ist nochmals zusätzlich die Zuverlässigkeit der Arbeit des Kopisten und die Echtheit der Urkunde in ihrer gesamten Diktion sowie Grafie auch für die Kopie erwiesen. Als Modernisierung ließe sich allenfalls das $<\mathrm{k}>$ für sonst im 11. Jahrhundert erwartbares $<c>$ ansehen.

Zusammenfassend und die Ausführungen in der „Geschichte der Stadt Leipzig“ im ganz bescheidenen Rahmen von Bemerkungen zur Existenz einer Siedlung Leipzig im 10. Jahrhundert sowie zum Namen des Burgwards ergänzend, kann von Folgendem künftig ausgegangen werden:

- Ein slawischer Siedlungsname war im 10. Jahrhundert bekannt, also bestand auch ein slawisch bewohnter Ort.

- Der Burgwardname beruht ebenfalls auf einer slawischen Bildung. Die Integration des Namens ins Deutsche ist urkundlich einmalig belegt 1050 und da bereits mit einer deutschen Deklinationsendung.

- Der Burgwardname und der ihm zugrunde liegende slawische Siedlungsname lassen auf eine bereits im 9., wenn nicht sogar schon im 8 . Jahrhundert vorhandene slawische Siedlung schließen.

- Die in der frühen urkundlichen Tradierung belegten Namen mit Lib- - also bei Thietmar von Merseburg Libzi und in der Urkunde von Kaiser Friedrich III. Libizken - zeigen Entlehnungen aus dem Slawischen mit dem Sprachgebrauch folgender korrekter Wiedergabe.

- Den slawischen Formen liegen zwei unterschiedliche Bildungen zugrunde, einmal als Örtlichkeitsname mit aus einer Personenbezeichnung *libbcb ,der Hagere" gebildetem Bewohnernamen *Lib'ci, die Hageren', der zum Siedlungsnamen wurde. Zum anderen der von der Basis des Ortsnamens abgeleitete Burgwardname *Libbsk-.

- Der zuerst bei Thietmar genannte Siedlungsname tritt noch mehrmals in der Überlieferung bis zum Ende des 12. Jahrhunderts entgegen: In der Corveyer ThietmarHandschrift als Libizi, in den Pegauer Annalen zu 1080 usque Libiz und 1089 oppidum Libiz ${ }^{21}$ sowie in einer Urkunde des Markgrafen von Meißen 1185 mit den Zeugen Albertus de Libz et frater suus Birzlaus. ${ }^{22}$

- Auch im 13. Jahrhundert finden sich nochmals zwei Lib-Schreibungen: 1212 apud Libuiz und 1219 apud Lybzeck sowie um 1225 Libzke. ${ }^{23}$

- Ab Ende des 12. Jahrhunderts ist augenfällig, dass der ursprüngliche Gebiets- bzw. Burgwardname mit $/ \mathrm{k} /$ im Auslaut nun als Ortsname neben die ältere Form tritt, was die zunehmenden Grafien mit $<\mathrm{k}>$ bzw. $<\mathrm{c}>$ [für gesprochen /k/ in lateini-

21 Walther, Der Name Leipzig (wie Anm. 1), S. 73.

22 CDS I/2 (wie Anm. 8), Nr. 510, S. 352.

23 Vgl. mit Quellenangaben HaNS WALTHER, Leipzigs Name im Lichte seiner Frühüberlieferung, in: Namenkundliche Informationen 95/96 (2009), S. 13. 
schen Texten] am Ende zeigen: 1190/95 neben Lipz auch Lipzk, 1216 neben Lipz wiederum auch Lipzc, 1217 Lipzc, 1222 Lipzk usw. ${ }^{24}$

- Mit der Bedeutungszunahme der von deutscher Seite als Burgwardmittelpunkt genutzten Siedlung und ihrer Burg wurde offenbar der in der deutschen Kanzlei und Verwaltung der Mark Meißen geläufige und vertraute Burgwardname mehr und mehr zur Kennzeichnung der Stadt verwendet und damit in dieser Form als Ortsname üblich.

- Die Verwendung der Formen Lipz mit Schreibvariante Lypz oder Lipcz/Lypcz ist noch bis ins 14. Jahrhundert anzutreffen. Diese einerseits wieder hinsichtlich des ursprünglichen slawischen Ortsnamens mit altem -c-Suffix (gesprochen [ts], geschrieben $\langle\mathrm{z}\rangle$ ), traditionellen Schreibungen bieten zugleich eine weitere Neuerung: Das ältere $<\mathrm{b}>$ wird zugunsten von $<\mathrm{p}>$ aufgegeben. Das ist eine geringfügige Veränderung in der Lautung des Namens, die am ehesten durch den deutschen Sprachgebrauch zu erklären ist und auf regressiver Assimilation beruht. Der in altsorbischer Zeit nicht mehr vorhandene ultrakurze $i$-Laut brachte /b/ und nachfolgend slawisch /c/ = [ts] in direkte Nachbarschaft. Die Stimmlosigkeit von [ts] bewirkte die Angleichung des vorangehenden stimmhaften /b/ zum stimmlosen /p/. Der Verlust der Stimmhaftigkeit des /b/ wurde dann in der Schrift mit $<\mathrm{p}>$ realisiert. Mitgewirkt haben kann dabei sowohl bei den slawischen Sprechern als auch bei den gebildeten deutschen Geistlichen in den Kanzleien die Kenntnis von slawisch lipa ,Linde' und die damit mögliche sekundäre semantische Verankerung des Namens als ,Lindenort'. Eigentlich hat der Ortsname damit auch für die Slawen eine - wenn auch neue - weiter durchschaubare Semantik und somit einen weiterhin verständlichen Inhalt erhalten.

- Die weitere sprachliche Entwicklung zur modernen Form Leipzig ist seit dem ausgehenden 12. Jahrhundert allein vom Deutschen bestimmt und geprägt worden. ${ }^{25}$

Der von Enno Bünz erstmals so klar erwiesene Charakter der Originalität der Urkunde von 1050 macht auch den von sprachhistorischer Seite bisher beobachteten Suffixwechsel in slawischer Zeit beim Siedlungsnamen in Gestalt einer rekonstruierten Form *Lip'sko o. ä. erstmals voll verständlich. Es hat sich klar herausgestellt, dass die Ursache in der Nutzung und Verwendung des Burgwardnamens zu suchen ist. In der Sprache der deutschen Obrigkeiten ist der Name für den Burgwardort dauerhaft fest und zum Namen der Stadt geworden.

Für die Sprachgeschichte und die Geschichte der Stadt Leipzig verdient noch ein weiterer Hinweis von Enno Bünz gesonderte Aufmerksamkeit. Mehr beiläufig erwähnt er noch eine Urkunde als „eine inhaltliche Fälschung vom Ende des 13. Jahrhunderts“ über eine Schenkung von Heinrich II. an das Bistum Merseburg von $1021^{26}$ mit der Angabe oppidum Libziki nominatum. Und er fügt hinzu, dass diese Urkunde als eine inhaltliche Fälschung aus dem 13. Jahrhundert „ohne Quellenwert für die frühere Zeit ist." 27 Zumindest unter sprachgeschichtlichem Aspekt besitzt diese Fälschung aber durchaus Quellenwert. Die Grafie mit $<\mathrm{b}>$ dokumentiert deutlich den im 13. Jahrhundert erfolgten Rückgriff auf eine ältere urkundliche Schreibung, vermutlich nach dem Vorbild einer älteren Urkunde, entsprechend der Lautung im 10., 11. und auch 12. Jahrhundert, während die Schreibung <ziki> bereits die jüngere deutsche und

24 Vgl. ebd., S. 15 f. mit noch weiteren urkundlichen Belegen vom 13. bis 15. Jahrhundert; ferner Walther, Zum Namen der Stadt Leipzig (wie Anm. 10), S. 134-140.

25 Vgl. dazu kurz gefasst Walther, Der Name Leipzig (wie Anm. 1), S. 73-76.

26 Monumenta Germaniae historica. Diplomata, Bd. 3: Die Urkunden Heinrichs II. und Arduins, Hannover 1900-1903, Nr. 528, S. 683.

27 Bünz, Herrschaftliche Strukturen (wie Anm. 12), S. 89. 
ab Ende des 13. Jahrhunderts sich allmählich durchsetzende übliche Auslautform mit den Grafien <zik> oder <zcik> bzw. <czig> und <zek> ausweist und damit wirklich Modernisierung - zumindest also partiell - bei der Fälschung bekundet. Das $-i$ im Auslaut in <ziki> wiederum dürfte auf Übernahme aus einer älteren Vorlage beruhen.

Damit ist die Schreibung vom Ende des 13. Jahrhunderts ad 1021 für den Sprachforscher durchaus von Aussagekraft: Einmal wird ein weiteres Beweisstück für die älteste Überlieferung des Leipzig-Namens mit der Basis <Lib $>$ geliefert, und zum anderen wird damit gleichzeitig mit <ziki> ein weiteres Mal die Fortführung des Burgwardnamens als Ortsname im 13. Jahrhundert mit nun vom deutschen Sprachgebrauch beeinflusster Suffixveränderung deutlich. Somit besitzt auch diese gefälschte Urkunde für die frühe Geschichte von Leipzig mit ihrer Schreibung sprachgeschichtlichen Quellenwert.

Hinzu kommt aber auch noch eine weitere positive Auswirkung aus den Ausführungen des Landeshistorikers zur Originalqualität der Urkundenkopie von 1050. Diese betrifft den Namen Wurzen. Der Ausstellungsvermerk lautet in nomine domini actum Wrcin und bringt damit die wohl älteste Aufzeichnung des Namens in seiner slawischen Lautung und in einer Form, die auf Übernahme ins Deutsche im 10. Jahrhundert hindeutet. Die Schreibweise reflektiert zweierlei, nämlich offenbar eine gute Kenntnis der slawischen Form beim Notar, wenn nicht sogar generell gute slawische Sprachkenntnis, und zugleich auch die Möglichkeit der Nutzung einer uns unbekannten älteren Vorlage oder aber die Bewahrung der Lehnform in althochdeutscher Zeit als etwa [wrtsin] mit einem silbisch gesprochenen $/ r /$ - also ziemlich exakt nach der späturslawischen Aussprache von *Vṛčbn- o. ä. Die Diffizilität der Etymologie des Namens hat der slavistische Sprachforscher Ernst Eichler wiederholt vor Augen geführt. ${ }^{28}$ Die historischen Zeugnisse des Namens lauten ab 961 (Kopie 11. Jahrhundert) Vurcine, bei Thietmar Vurcin sowie Vurzin und geben damit bereits die im Altsorbischen eingetretene Entwicklung des silbischen $r$-Phonems zu Vokal plus - $r$ - wieder. $\mathrm{Ob}$ der Name direkt erst in slawischer Zeit gebildet wurde, lässt sich allerdings nicht mehr entscheiden. Es ist durchaus möglich, dass eine vorslawische Form slawisiert wurde, wie auch der russische Gewässername Vorskla sehr wahrscheinlich eine slawische Eindeutung zu einem vorslawischen Hydronym darstellt und dabei an die auch im Namen Wurzen zu vermutende slawische Wurzel angeschlossen wurde. ${ }^{29}$ Der russische Gewässername Vorčenka im Oka-Gebiet dürfte eine originäre slawische Bildung sein. ${ }^{30}$ Die slawische Wurzel ist auch in der südslawischen Hydronymie vertreten, vgl. slowenisch Vrčica. ${ }^{31}$ Es bleibt aber trotz dieser Vergleichsnamen ungewiss, welches Motiv dem heutigen Ortsnamen Wurzen ursprünglich zugrunde liegt sowie auch, ob eine appellativische oder anthroponymische Verbindung in slawischer Zeit wirksam geworden ist. ${ }^{32}$

Der in der "Geschichte der Stadt Leipzig“ insgesamt doch nur wenige Zeilen ausmachende Vermerk des Landeshistorikers Enno Bünz zur Zuverlässigkeit der Urkunde aus dem Jahr 1050 und Erwähnung des Burgwards mit den Worten in

28 Vgl. im Überblick dazu Ernst Eichler, Die slawischen Ortsnamen zwischen Saale und Neiße. Ein Kompendium, Bd. 4, Bautzen 2009, S. 91.

29 Vgl. Oleg Nikolaevič Trubačev, Nazvanija rek pravoberežnoj Ukrainy [Gewässernamen der Ukraine], Moskva 1968, S. 138 mit weiterer Literatur.

30 Vgl. Galina Petrovna Smolickaja, Gidronimija bassejna Oki [Hydronymie des Oka-Einzugsgebietes], Moskva 1976, S. 97.

31 Vgl. France BezlaJ, Slovenska vodna imena, II. del, Ljubljana 1961, S. 316.

32 StanisŁaw Rospond, Slawische Namenkunde, Heidelberg 1989, S. 126 verweist bei vrč ,Krug, Becher' auch auf mögliche metaphorische und metonymische Motivik. 
burgvardo Libizken hat für den Sprachhistoriker doch eine ganze Reihe von neuen Einsichten möglich gemacht:

- Die Aussage von Enno Bünz, „Der Burgward Leipzig muss 1015 bereits einige Zeit bestanden haben“, 33 ist aus sprachgeschichtlicher Sicht voll zutreffend und zu bestätigen. Die sprachgeschichtlichen Fakten erfordern regelrecht die Existenz des Burgwards bereits im 10. Jahrhundert, sonst wäre eine Form wie 1050 in burgvardo Libizken nicht möglich gewesen.

- Libizk- muss vor der Jahrtausendwende schon von der deutschen Obrigkeit gebraucht worden sein. Wäre der Burgwardname erst nach 1000 n. Chr. auf slawischer Basis übernommen bzw. verwendet worden, hätte er die grafische Gestalt *Libzk-, mit althochdeutscher Lokativendung dann *Libzken, erhalten, also somit dann adäquat zu der Aufzeichnung der Form Libzi bei Thietmar für den Ort mit Burg und Kirche.

- Die Schreibungen in den Urkunden und Quellen generell sind also gerade bei den historischen Formen des heutigen Ortsnamens Leipzig wie auch schon des Burgwardnamens als sehr zuverlässig zu bewerten.

- Die Burgwardangabe mit Libizken war bisher als völlig entgleiste Schreibung angesehen worden. Sie passte einfach nicht in die Traditionslinie der Überlieferung, weil sie als Fälschung aus dem 15. Jahrhundert bzw. als Schreiberform von um 1430 aufgefasst wurde. Durch die Korrektur zur verlässlichen Datierung der Urkunde zu 1050 und die sprachwissenschaftlich erwiesene recht originalgetreue Abschrift hat sich das Bild völlig gewandelt. Die Schreibung von 1050 entspricht voll der altdeutschen Zeit und dem damaligen Sprachgebrauch. Damit bestätigt die ganz einmalige sprachliche Form Libizken zugleich die Beibehaltung einer Sprachgestalt aus dem Original bei späterer Abschrift. Eine wirkliche Modernisierung im 15. Jahrhundert hätte etwa Lipziken ergeben müssen, niemals aber die Grafie mit $<$ bizk> aufweisen können.

Resümierend lässt sich immerhin konstatieren: Wo die berichtenden Quellen mit ihren Aussagen für den Historiker enden, vermag der Sprachforscher auf der Basis solcher gesicherten Quellen noch mit „Sonden“ in Zeiträume vor der verschriftlichten Überlieferungszeit zu gelangen und auf diesem Weg gewissermaßen „verborgene“ geschichtliche Fakten zu ermitteln. Damit wird am untersuchten Gegenstand wiederum deutlich, wie nötig und ertragreich sowie wechselseitig bereichernd transdisziplinäre Forschung sein kann.

Es könnte nun vielleicht der Eindruck aufkommen, dass die Schreibungen in der Zeit nach der fränkischen Eroberung des ostsaalischen Gebietes hier überbewertet werden. Dem ist entgegenzuhalten, dass Schreibungen für die Identifizierung und Orientierung im geografischen Großraum gerade im Mittelalter sehr wichtig waren. Das macht die Zuverlässigkeit der Grafien ursprünglich slawischer und auch deutscher Namenformen aus. Knapp zusammengefasst ergibt sich zu den A u f z e i c h n u n ge n der Vorgängerformen des heutigen Namens Leipzig34 folgende die Entwicklung im Deutschen illustrierende Übersicht:

- Libiz-als Lehnname für die slawische Siedlung aus dem 10. Jahrhundert mit Aufzeichnungen bis ins 12. Jahrhundert;

- Libizk- als Lehnname für den Burgward seit dem 10. Jahrhundert;

33 BüNZ, Herrschaftliche Strukturen (wie Anm. 12), S. 82.

34 Grundlage sind die von Hans Walther zusammengestellten Belegreihen in: Leipziger Stadtgeschichte. Jahrbuch 2009, S. 14-16; und in: Namenkundliche Informationen 95/96 (2009), S. 12-14. 
- Libz-als Lehnname im 11. und 12. Jahrhundert für den slawischen Ort mit Burg und Kirche - also Neuentlehnung aus der nach $1000 \mathrm{n}$. Chr. lautlich veränderten altsorbischen Form; ebenso 1021 Libziki als Fälschung im 13. Jahrhundert mit Bewahrung von <Libz $>$ wohl infolge von Übernahme aus einer älteren Vorlage;

- Lipz mit grafischen Varianten ab Ende des 12. Jahrhunderts bis ins 14. Jahrhundert für den Ort mit lautlich $/ b />/ p /$ als Veränderung im Deutschen;

- Lipzc/Lipzk als deutschsprachige Fortführung des ursprünglichen Burgwardnamens, nun für den Ort bzw. die inzwischen entstandene Stadt ${ }^{35}$ gebraucht, vom Ende des 12. bis Ende des 14. Jahrhunderts;

- Lipzic/Lipzik mit Variationen ${ }^{36}$ setzt sich ab Ende des 13. Jahrhunderts für die Stadt zunehmend durch und beruht auf Angleichung an den im Deutschen geläufigen Auslaut auf -zig, vgl. schließlich 1500 Leipzig.

Beachtenswert ist, dass die ursprüngliche inhaltliche Bedeutung der Namenbasis Lib-, also die etymologische Seite, bei der Namenverwendung keine Rolle spielte. ${ }^{37}$ In der Zeit des Nebeneinander und Miteinander von deutschen Herrschaftsträgern und slawischer Bevölkerungsmehrheit war ausschließlich die für beide Ethnien gleichermaßen verständliche Verwendung von Eigennamen zur Sicherung der Identifizierung der einzelnen Objekte unabdingbar. Daher ist vom 10. bis zum 12. Jahrhundert die Überlieferung der zunächst älteren Libiz-Formen - etwa im Sinne von „Lib-Ort“38 _ und vom 11. Jahrhundert an auch der Libz-Formen als besonders aufschlussreich zu beachten. Erst vom Ende des 12. Jahrhunderts an mit dann zunehmend mehrheitlich deutschen Bewohnern setzt das Aufkommen der Lipz- und der Lipzk-Grafien ein. Aber nur für die des Slawischen Kundigen konnte der Name nun mit der Semantik ,Linde“ verbunden werden.

Insgesamt bietet die Geschichte des Namens der Stadt Leipzig eine durchaus komplizierte Entwicklung. Zugleich liefert die historische Tradierung des Namens eine sehr aufschlussreiche Dokumentation. Der Sprachhistoriker vermag beim heutigen Forschungsstand einen primär slawischen Namen für eine erste Ansiedlung zu ermitteln. Auf der Basis des Siedlungsnamens aus dem 8. oder 9. Jahrhundert ist in sprachgeschichtlich nicht genau bestimmbarer Zeit ein neuer slawischer Name gebildet worden, der als Burgwardname verwendet wurde. Die slawische Siedlung wurde zum Zentrum für das umliegende Gebiet und besaß im 11. Jahrhundert die bei Thietmar bezeugte Burg und kirchliche Einrichtung. Der seiner Herkunft nach slawische Burgwardname muss aufgrund seiner Lautung bzw. frühen schriftlichen Überlieferung ebenso wie der slawische Siedlungsname bereits im 10 . J a h rh undert in den deutschen Sprachgebrauch übernommen worden sein. Der Siedlungsname setzt folglich einen $\mathrm{O} r \mathrm{t}$ als in jener Zeit e $\mathrm{x}$ is $\mathrm{t}$ e $\mathrm{n} \mathrm{t}$ voraus.

35 Um 1225 Libzke (neben Lipzke) lässt erkennen, dass die ursprüngliche Lautung mit /b/ bzw. Schreibung mit $<$ b $>$ immer wieder nachwirkt. Das zeigt sich auch später nochmals: um 1285 Libziki als der bislang letzte Beleg mit $<\mathrm{b}>$.

361219 apud Lybzeck ist der bisher älteste Beleg für die Auslautangleichung an deutsch -zig.

37 Für die frühen Siedler besaßen vorgefundene und damit im neuen Siedlungsraum bereits verwendete Namen entscheidende Relevanz als Orientierungshilfe. Dabei spielte die den Namen innwohnende etymologische Bedeutung keine Rolle. Vgl. dazu auch die zutreffenden Ausführungen zum Maingebiet bei Harald Bichlmeier/ Wolfgang Vorwerk, Der Gewässer- und Ortsname LOHR: ein unlösbares Problem?, in: Beiträge zur Geschichte der Stadt und des Raumes Lohr 4 (2015), S. 50.

38 Das slawische Suffix leistete dabei dasselbe mit Bezug auf den zugrunde liegenden Gebietsnamen wie in unserer Zeit der Ortsname Darßer Ort im Anschluss an den Gebietsnamen Darß. 
Der Name für den Burgwardort als eindeutige Lehnform aus althochdeutscher Zeit beweist in seiner Struktur die bereits im 10. Jahrhundert vollzogene Entlehnung aus dem Slawischen. Eine im 11. Jahrhundert vollzogene Neuentlehnung des Siedlungsnamens war bedingt durch die eingetretene lautliche Veränderung des Namens bei den Slawen der Region. Die neue Lehnnamenform sicherte die eindeutige interethnische Kommunikation und folgte der sprachlichen Entwicklung im slawischen Umland.

Wenn nun sichtbar und nachvollziehbar geworden ist, dass sprachgeschichtliche Forschungsergebnisse immer erst auf exakter historischer Faktenbasis möglich werden, dann aber wiederum durchaus zur Erweiterung unseres Wissens über geschichtliche Zeiträume vor den ersten schriftlichen Aufzeichnungen von Nachrichten zu einem bestimmten Gebiet führen können, ist das eigentliche Ziel dieser Wortmeldung erreicht. Die wechselseitige Bereicherung und Gewinnung oder auch nur Vertiefung neuer Erkenntnisse ist durch die Kooperation von Landes- und Sprachgeschichtsforschung auch weiterhin wünschenswert. Der Landeshistoriker Enno Bünz hat mit seinen Aussagen und Forschungsergebnissen zur urkundlichen Überlieferung für die Namenforschung drei ganz entscheidende, bereichernde und weiterführende Hinweise gegeben:

1. Er hat die Originalität des Inhalts der nur in Abschrift vorhandenen Urkunde von 1050 erwiesen und sie damit zu einer zuverlässigen Quelle für die Sprachforschung erklärt. Letztere hat zusätzlich auch die Form, speziell die Schreibung der geografischen Namen in der Kopie, als dem Original folgend und weitestgehend entsprechend geklärt.

2. Er hat die bisherige Annahme, bei der Urkunde von 1050 handle es sich um eine spätere Fälschung aus dem 15. Jahrhundert widerlegt und damit auch die bisher von der Sprachforschung eingenommene Position hinfällig gemacht.

3. Er hat damit die Voraussetzung geschaffen, sowohl a) die für das 15. Jahrhundert absolut abwegige Schreibform Libizken sprachgeschichtlich neu beurteilen zu können als auch b) den bisher etwas ungewöhnlichen slawischen Suffixwechsel in der älteren Geschichte des Ortsnamens Leipzig von -bci zu -bsk-als Folge der Verwendung des ursprünglichen Lehnnamens für den Burgwardort schließlich ab Ende des 12. Jahrhunderts als Name für den Ort bzw. die Stadt generell erklären zu können.

Die Lektüre in Band 1 der „Geschichte der Stadt Leipzig“ erwies sich für die Geschichte des Leipzig-Namens als sehr ertragreich. Die sprachlichen Zeugnisse für eine slawische Siedlung als Vorgänger der Stadt Leipzig sind nochmals stichwortartig angeführt worden. Zweifel an der Existenz einer slawischen Siedlung seit dem 8./9. Jahrhundert oder auch nur Vorbehalte dürften nun eigentlich verstummen. Und der Burgwardname ist auch zweifelsfrei als eine slawische Bildung aus dem 10. Jahrhundert erwiesen.

Es bleibt zu hoffen und zu wünschen, dass die absichtlich etwas ausführlich ausgebreiteten Zusammenhänge zwischen einem Namen, seiner Geschichte und seinem Quellenwert sowie den Schlussfolgerungen für das den Namen tragende reale Objekt und sein Alter auch seitens der Geschichtsforschung Akzeptanz finden werden. Nur dann wird wohl interdisziplinäre Forschung erst ertragreich und lässt sich auch als solche wirklich bezeichnen. 